\title{
SINUS BRADYCARDIA WITH CARDIAC ASYSTOLE
}

\author{
BY

\section{R. S. BRUCE PEARSON}

From King's College Hospital

Received December 22, 1944

The case history of a patient suffering from attacks of cardiac asystole with syncope is described. On superficial examination the condition appeared to be one of Stokes-Adams disease; electrocardiographic tracings, however, showed that there was no delay or block in the conducting system of the heart, but a persistent sinus bradycardia (about 24 beats a minute) interrupted by complete asystole for periods of at least 22 seconds. No report of a similar case has been found. The effect of exercise and various drugs upon the heart rate is described and the underlying basis of the condition discussed.

The patient was a woman of fifty-two years. She had led an active life until the onset of the present symptoms. Apart from excessive menstrual loss for nine years after the birth of her child, she had no history of previous illness. The menopause took place a year before admission.

In February 1943 she first complained of occasional dizziness, particularly after stooping. At this time she was found to have a regular pulse rate of 24 beats a minute. These attacks became rather worse two months before she was first seen in December 1943. During this month while in her garden she had her first attack of loss of consciousness. She felt faint and fell to the ground before she was able to sit down. Three weeks later she lost consciousness for the second time just after a cardiogram had been taken. Following this she had two or three attacks weekly until her admission to hospital on 29/1/44.

At this time her pulse was forceful and regular at 24 beats to the minute. The heart sounds were normal, the blood pressure somewhat raised (195/105). Radiographic examination of the heart showed slight generalized enlargement. There was no evidence of arteriosclerosis.

Shortly after admission it was observed that her pulse was not always regular; additional beats were often present, sometimes occurring singly and sometimes in short runs of two or three. She felt faint frequently and was aware that her heart had ceased to beat or was beating irregularly. At times she lost consciousness: sometimes this happened without her knowledge, for example, on several occasions she realized that she had fainted only because the cup she had been holding had fallen from her hand; usually she was distressingly aware of imminent loss of consciousness. Her description of her sensations at these times was as follows: "In very mild attacks there is a feeling of dizziness causing me to stand still if I am walking and a feeling as if my body was leaving me." In more severe attacks " there is a feeling as if cotton wool was being rammed into my arms and as if my arms and legs were in clamps." During these attacks she felt very frightened.

Observation of these attacks, which were now occurring very frequently, confirmed the absence of pulse at the wrist during them, and on auscultation no heart sounds could be detected. The pause, which might last for from 5 to 22 seconds, was usually followed by several rapid, irregular beats before the normal rate of 24 beats a minute was resumed. Tracings during these attacks showed that there was complete standstill of the heart followed by an increased rate, partly due to extrasystoles. During the attack the patient's face became pale and then flushed as the pulse returned, so that even minor attacks could easily be recognized by observation from the end of the bed. In more prolonged periods of asystole consciousness was lost. In these, after the preliminary pallor, a look of fearful expectation would appear, followed by movement of the head and eyes to the right with clenching and sometimes grinding of the teeth and swallowing movements; at times she would take a sighing inspiration, the arms being flexed at the elbows and the hands raised in a series of jactitating contractions of the forearms and clutching movements of the hands. It was difficult to tell at what stage consciousness was lost, but on several occasions the patient continued to talk for at least seven 
seconds after the pulse had become impalpable, before she stammered and ceased speaking. On three occasions standstill was observed to last for 22 seconds. After long pauses the cardiac contractions were especially vigorous and the head and shoulders were shaken by each pulsation which could be clearly seen in the carotid vessels. At the same time a deep flush spread over her face.

The patient thought that the attacks were worse after effort and particularly noticed that the use of a bed-pan or even talking in an animated way precipitated them. The frequency with which they occurred when she was being examined suggested the possibility of an emotional precipitating factor. Air raids, including the dropping of heavy bombs in the vicinity, had no effect except on one occasion. The patient was then aware that her heart had suddenly started to beat more rapidly, and the night nurse reported that the rate rose to 100 beats a minute and continued at this level for ten minutes but then returned to 20 beats a minute; this was the only occasion at rest under natural conditions when a rate greater than 30 beats a minute was recorded. Although she usually slept well at night, irregular beats and long pauses were noticed during sleep by the night nurse.

At times she would have a series of attacks, being scarcely out of one before the next took place, over a period of several hours. Sometimes she vomited during these bad periods. On 27/2/44 she was examined while having a series of attacks following a good night. The first occurred while the patient was on the bed-pan. When seen later the pulse was irregular, with periods of standstill up to 10 seconds followed by irregular and often coupled beats. The face was flushed and became pale repeatedly. The patient vomited several times. On 12/3/44 she was observed for forty-five minutes during which at least fifteen attacks occurred and on one occasion three periods of asystole lasting from 7 to 9 seconds took place in one minute, the first with loss of consciousness. During the last three months of her stay in hospital the attacks became much less frequent and usually followed exertion.

After her discharge from hospital in August 1944, she continued to have frequent faint turns and lost consciousness several times weekly. At this time she was staying with friends away from home and was worrying about her husband and daughter. For three weeks before she returned home at the end of October she had no loss of consciousness, although she was aware of short pauses, double beats, and runs of four or five irregular beats. On 29/10/44 when she was examined her pulse rate was in the region of 20 and periods of coupling were frequent when only alternate beats got through to the wrist. On this day the blood pressure when first taken was 230/110, but after an hour the systolic pressure had fallen to $180 \mathrm{~mm}$. The Wassermann reaction was negative. The blood sugar during a series of attacks was within normal limits. The serum potassium was $21^{\circ} 6 \mathrm{mg}$. per 100 c.c.

Electrocardiographic tracings were taken on a number of occasions. These showed that the basic rate varied between 18 and 24 . The complexes showed normal sinus rhythm. There was constantly a large $\mathrm{T}$ wave, particularly in the second lead. Periods of cardiac standstill were observed up to 9 seconds and were followed by a rise in rate to 50 or more for a few seconds. Exercise had a similar effect. Impulses frequently occurred in pairs from the S-A node, but on other occasions the coupling was shown to be due to auricular or ventricular extrasystoles. Isolated auricular and ventricular extrasystoles were often observed. There

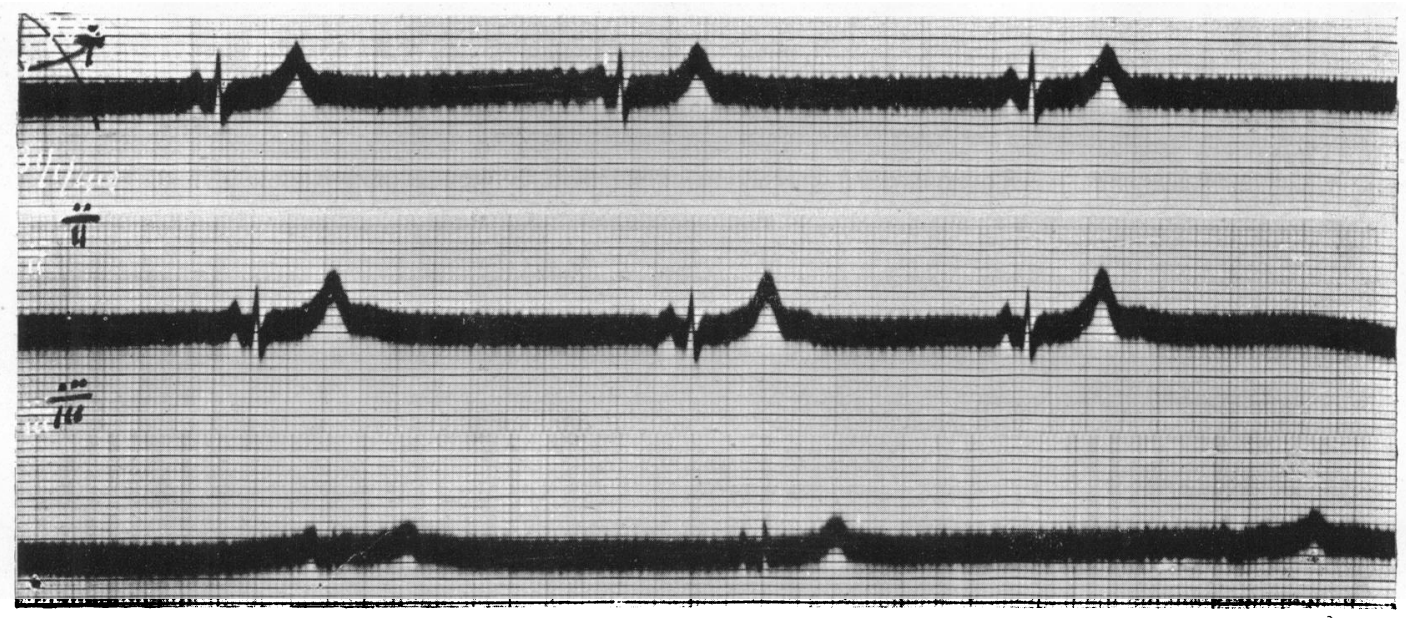

Fig. 1.-Electrocardiogram showing her usual basic rate of 24 a minute. All figures have been slightly reduced in size $(43 / 50)$. 


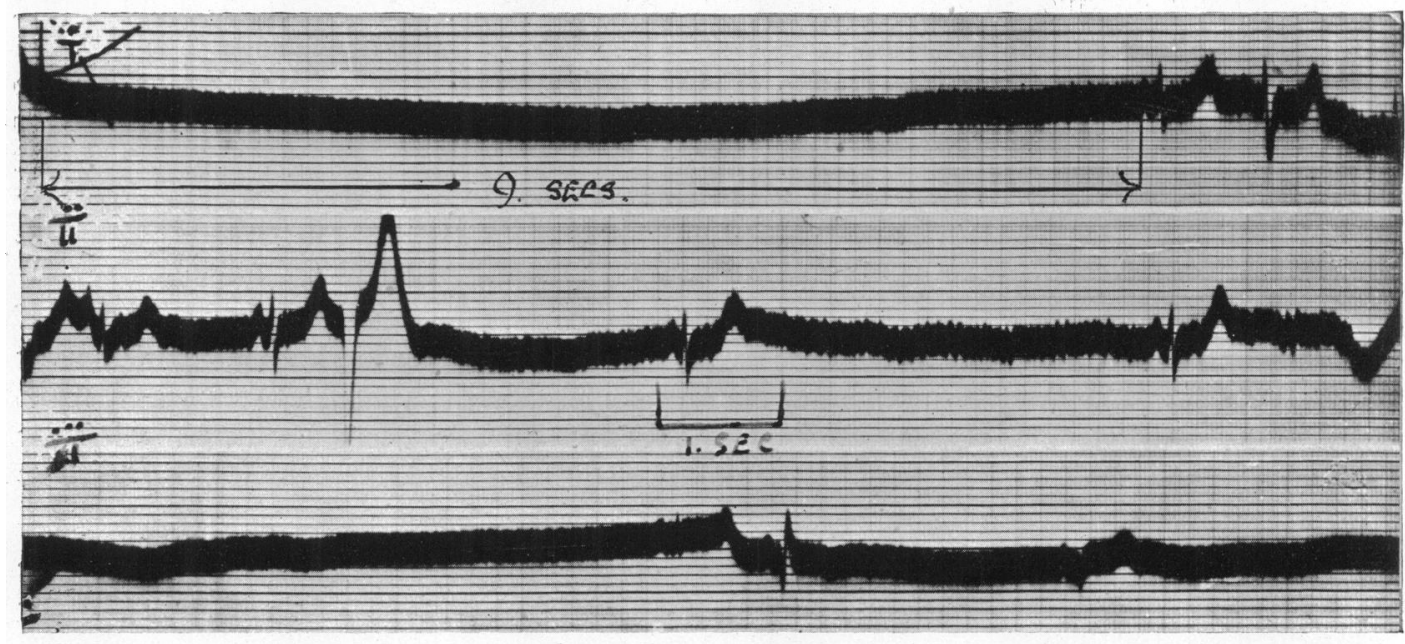

FIG. 2.-Asystole for 9 seconds in lead I. Lead II shows a ventricular extrasystole; lead III shows the end of a further period of asystole.

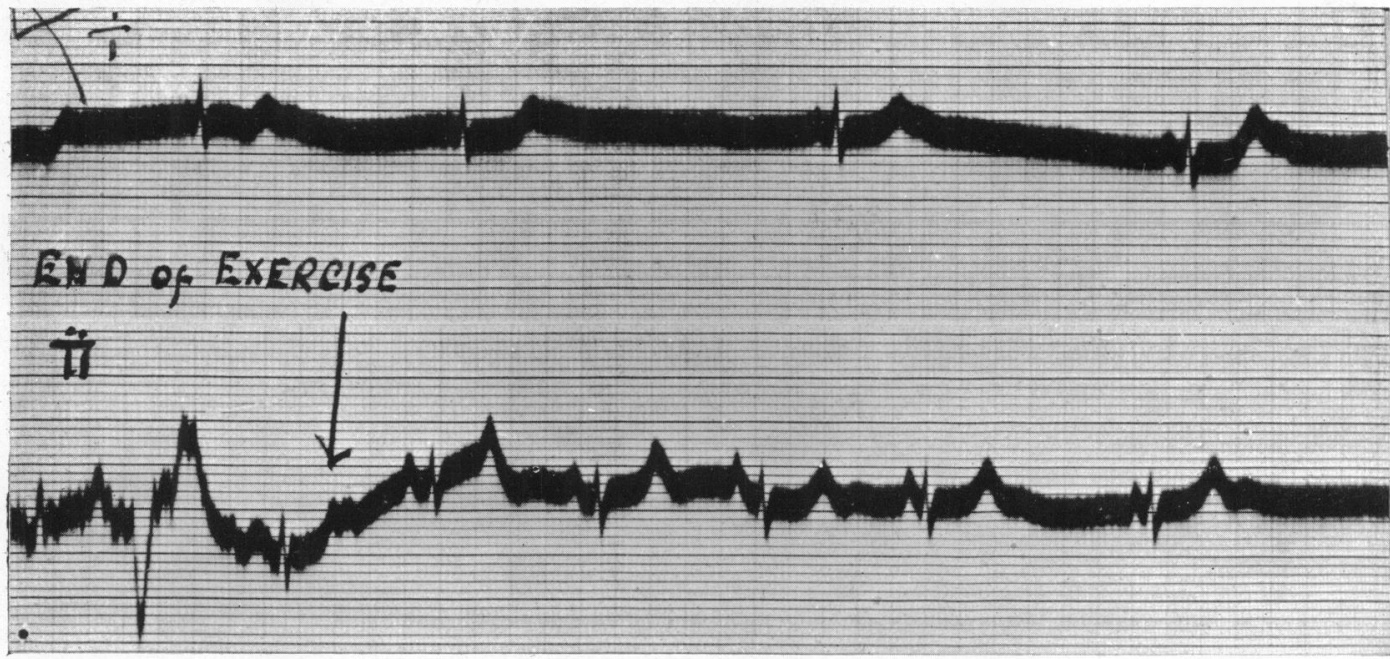

FIG. 3.-The increase of rate caused by exercise is shown in lead II.

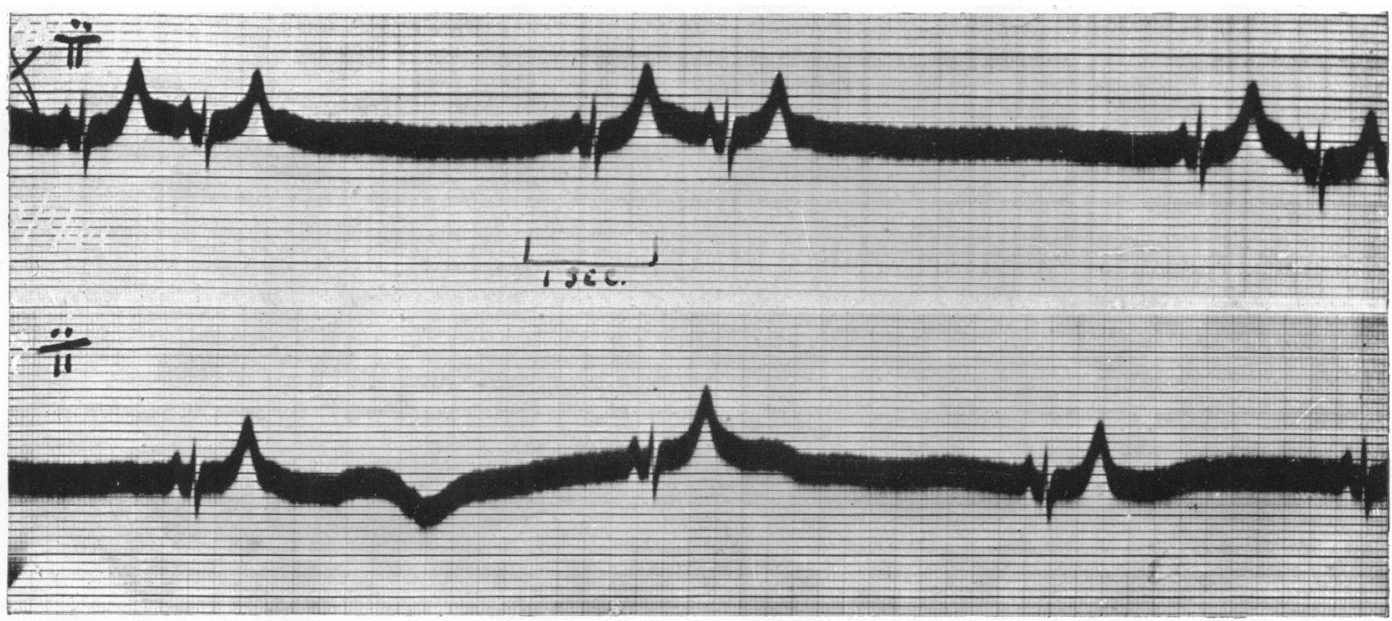

Fig. 4.-Coupled beats, the second being a premature beat arising from the sinus. 
was no delay in conduction and no evidence of nodal escape or of ventricular rhythm during periods when the sinus failed.

On 22/6/44 a cardiographic tracing was recorded continuously for about half an hour, with a simultaneous electro-encephalogram. During this period every abnormality seen in previous cardiographic tracings was recorded. At times a regular rate between 18 and 24 beats each minute was observed. Premature beats giving rise to coupling at regular intervals sometimes occurred on from five to ten successive occasions, the extrasystole sometimes arising repeatedly from the ventricle, sometimes from the sinus or the auricle, and sometimes from varying sites after successive normal beats. On one occasion a period of asystole up to 12 seconds was followed by 15 ventricular extrasystoles in 11 seconds, on another 14 beats arising sometimes from the auricle and sometimes the ventricle were recorded in 10 seconds. Periods of asystole occurred frequently: on two occasions these lasted for 13 seconds, and once for 12 seconds. Asystole of 10-11 seconds occurred twice, 8-9 seconds three times, 7-8 seconds ten times, 6-7 seconds twelve times, 5-6 seconds thirteen times, and 4-5 seconds ten times. Such periods of asystole were followed by single normal beats, coupled beats, short runs of extrasystoles, or irregularly spaced beats with occasional ventricular extrasystoles.

The following example shows the extreme variations that could occur consecutively in 43 seconds-asystole for 7 seconds, a coupled beat, both impulses arising from the S-A node, an interval of 2.5 seconds followed by a normal beat and a ventricular extrasystole, an interval of 3 seconds, a normal beat followed by a premature auricular beat, asystole for 5 seconds, a normal beat followed by two ventricular extrasystoles in close succession, asystole for 5 seconds, a normal beat with a premature ventricular beat, asystole for 5 seconds, a coupled beat, both impulses arising from the S-A node, asystole for 4 seconds followed by another coupled beat from the S-A node, an interval of 3 seconds followed by a further coupled beat.

The electro-encephalogram, which was recorded simultaneously, was entirely normal except that after a period of asystole there were minimal change in rhythm due to cerebral anoxæmia. This investigation was carried out by Dr. J. D. N. Hill, for whose help I am grateful.

The following observations were made.

Effect of exercise. This was always followed by an increase of pulse rate for some seconds, but this was usually succeeded by periods of asystole. Very slight exertion caused extrasystoles or coupling and the pulse was always markedly irregular on these occasions. Rising from a lying to a sitting position ten times caused a very transient rise to 60 beats a minute. If the patient held her breath for 15 to 30 seconds similar irregular pulsation with gaps would occur,

Compression of carotid sinus. This had absolutely no effect on the pulse rate or blood pressure on a number of separate occasions when it was tried.

Effect of atropine. 1/50 of a grain of atropine was injected intravenously on 8/2/44. The pulse rate before the injection was regular at 20 a minute. After ten minutes the pulse rate had risen to 27 , after fifteen to 31 , after thirty to 31 , after forty-five to 33 . The mouth was then dry and the pupils widely dilated. The pulse rate remained at 33 to 34 until three hours after the injection and then gradually fell.

On $19 / 5 / 44,1 / 25$ of a grain of atropine was given intravenously. The pulse rate was regular at 20 before the injection. After five minutes it had risen to 34 beats at the apex. These were coupled and only 17 were counted at the wrist. This coupling continued for fifteen minutes. After twenty minutes the rate was regular at 32 at the wrist and apex. The vision was blurred and the mouth dry: after an hour the rate had risen to 34 and was regular. It continued at this height for several hours, but six hours after the injection had fallen to 25 .

It was noticed that the blood pressure, which was $168 / 90$ before the injection, rose to $212 / 90$ after half an hour, to $208 / 90$ after forty minutes, and had fallen to $190 / 90$ after two and a half hours. As a result of this injection the patient suffered from transient mild symptoms of atropine poisoning and was hallucinated and disorientated.

Atropine in full doses, therefore, caused a slight increase in rate with a regular pulse. Medicinal doses (1/100 of a grain and regular heavy doses of belladonna) given when the patient was having frequent attacks of faintness had no obvious beneficial effect. The patient thought that periods of asystole were rather more frequent during this treatment:

Effect of injection of adrenalin. 5 minims of adrenalin was injected subcutaneously on 
$4 / 2 / 44$. The pulse rate before the injection was 20 . It rose to 27 after twenty minutes and was somewhat irregular at this time due to extrasystoles. On 29/10/44 the pulse was regular at 19 at the wrist, but with coupled beats audible at the apex for fifteen minutes before 5 minims of adrenalin were given. After five minutes the patient was trembling slightly but the pulse rate was unaltered and the beats were still coupled; after seven minutes 22 coupled beats were counted, after nine minutes 28; at eleven minutes the pulse became irregular with asystole of seven seconds followed by six or seven rapid beats. No further rise in rate occurred but the pulse became more irregular and periods of asystole of 5-7 seconds occurred once or twice each minute until half-an-hour after the injection, when the pauses ceased, coupled beats diminished, and a regular rhythm of 24 beats to the minute was observed twenty-seven minutes after the injection. Occasional coupled beats were felt after this but after 42 minutes the rate was regular at 28 , and after 45 at 24 a minute.

Adrenalin, therefore, appeared to produce an effect resembling that of exercise, leading to a slight increase of rate, with periods of asystole.

Effect of ephredrine. Ephedrine, 1/2 grain given by mouth on 10/2/44, was apparently followed by some increase in the number of extrasystoles, although the pulse rate, which was 19 before administration, never rose to more than 22 during the next hour.

Effect of thyroxin. Thyroxin, $2 \mathrm{mg}$., was given intravenously on 7/5/44. The pulse rate beforehand was 18 and regular; blood pressure 152/80. After ten minutes the pulse rate was 16 and regular; after twenty minutes, 16 regular; after thirty minutes, 19 with three extrasystoles, systolic blood pressure 156; after forty minutes the pulse rate was 17 with 4 coupled beats. After an hour the pulse rate was 19 with 3 coupled beats; after 2 hours, 20 with 3 coupled beats; after 3 hours, 22 and regular. The rate continued between 23 and 24 for a number of hours. This dose of thyroxin had no obvious effect on the pulse rate.

The administration of acetylcholine or its derivatives was considered too dangerous owing to the possibility of increasing the periods of asystole. Their effect was not, therefore, observed.

The following drugs were given therapeutically. Tinct. Belladonna, up to 20 minims, fourhourly, had no obvious effect on the pulse rate but made the patient dry and uncomfortable; during the time she was on this preparation she had many faint turns. Injection of atropine, 1/100 of a grain, was likewise ineffective in preventing attacks of asystole. Thyroid sicca, 1/4 of a grain t.i.d., had no effect. Barium chloride, 1/2 a grain t.i.d., Luminal, $1 / 2$ a grain t.i.d. by mouth, Nicotinic acid, $100 \mathrm{mg}$. daily, and Benerva, $50 \mathrm{mg}$. daily, given intravenously, had no obvious effect on the pulse rate. It was thought that the atropine preparations and the thyroid possibly led to an increase in the irregularity of the pulse and in the number of faint turns.

\section{Discussion}

Loss of consciousness with disappearance of the pulse at the wrist and absence of heart sounds is commonly due to one of three clinical conditions-simple fainting resulting from vaso-vagal disturbances, heart block (Stokes-Adams disease), or stimulation of the vagus nerve as the result of a sensitive carotid sinus reflex or more rarely by pressure from a tumour or aneurysm. The patient whose history has been recounted did not suffer from any of these conditions. Simple fainting is unlikely to occur at rest in bed, particularly during sleep, nor were the usual prodromal symptoms of salivation and perspiration ever recorded; there was no cardiographic evidence of heart block; the symptoms could not be reproduced by pressure over the carotid sinuses, nor were they prevented by atropine. Moreover, none of these conditions offers any explanation of the persistent bradycardia (varying between 15 and 30 beats a minute) known to have been present for seven months while the patient was in hospital and probably for at least two years.

Other rare causes of cardiac syncope have been described. In sino-auricular block, Type A (Cowan), the sinus stimulus may default so that auricles and ventricles miss one or more beats, and fainting may occur. The interval between successive beats is in this case always a multiple of the normal beat, i.e. it may be two, three, or four times the normal interval between auricular contractions. The pulse rate in S-A block is usually within normal limits, 
but Cowan records the history of a man, aged 63, who suffered from syncopal attacks and had a basic heart rate of 30 due to a sinus bradycardia. This was thought to be the result of impaired blood supply of the S-A node following coronary infarction. Although this case is in many ways similar to ours, the absence of any regular mathematical relationship between the periods of asystole and the preceding pulse rate excludes sino-auricular block in the latter.

Laslett (1908-09) described the case of a woman, aged 40, suffering from fainting attacks due to arrest of the whole heart, as demonstrated by sphygmographic tracings of the jugular pulse. In this patient the basic rate was 60-70 beats a minute. Unconsciousness occurred if asystole lasted for more than 7 seconds. The irregularity of the intermissions in this case excludes S-A block. After atropine, 1/50 of a grain subcutaneously, the pulse rate rose to 100 and remained regular for a number of hours: exertion or excitement also temporarily prevented periods of asystole. Laslett concluded that increased vagus influence was responsible for the failure of the S-A node in this case.

Wedd also records the history of a boy, aged 16 years, complaining of fainting, in whom the heart rate varied between 32 and 86 with periods of standstill of from 3-7 seconds. The electrocardiogram showed, however, that the heart beat originated from the A-V node. This nodal rhythm persisted after atropine (1/33 of a grain), but periods of asystole were abolished and the pulse rate rose from 40 to 86 . The periods of cardiac standstill appear to have been due to excessive vagal stimulation.

Trocmé (1922) describes two cases of sinus bradycardia with no increase of rate following atropine, and attributes the condition to hypo-excitability of the S-A node. Periods of asystole occurred, which he attributed to increased vagal tone.

This patient differs from most others with cardiac standstill due to rare disturbances of rhythm in the failure of atropine to abolish the periods of standstill or to raise the basic pulse rate to a normal level. This striking ineffectiveness of stimulation or paralysis of the vagus nerve to modify the heart's action suggests that the S-A node is less excitable than normal. The slight effect of exercise and other drugs such as adrenalin, thyroid, or barium chloride is further confirmation of this view. Trocmé's cases alone may have had a mechanism similar to ours.

The reason for the development of a condition of such reduced excitability remains obscure. There was some evidence that psychological factors might influence her condition, but it is unlikely that the condition could be explained on this basis. In view of the patient's age, diminution of the blood supply to the region of the pacemaker as a result of atheroma seems to be the most probable cause. On the other hand the failure of the A-V node or of the ventricle to initiate their own rhythm during periods of prolonged asystole may indicate a general condition of hypo-excitability.

\section{SUMMARY}

A case of persistent sinus bradycardia with syncopal attacks due to cardiac standstill is described.

Evidence is given for believing that this is not vagal in origin but due to hypo-excitability of the pacemaker.

I should like to thank Dr. Terence East and Dr. Parkinson for their helpful advice.

\section{REFERENCES}

Cowan, J. (1939). Brit. Heart J., 1, i.

Laslett, E. E. (1908-09). Quart. J. Med., 42, 347.

Wedd, A. M., and Wilson, D. C. (1929-30). Amer. Heart J., 5, 493.

Trocmé (1922). Etude des Accidents Syncopaux au cours des Arrhythmies. Paris, quoted by Wedd and Wilson. (I have not been able to consult this reference.) 